\title{
Long disease-free survival (48 months) in a breast cancer patient with ovarian and pelvic metastasis - a case report
}

\author{
Yu Feng ${ }^{1,2}$, Xia Zhao ${ }^{1}$, Shuzhen Lv $^{1}$, Bingjun Xiong ${ }^{1}$, Yanping Li $^{1}$, Man Zhang ${ }^{2}$ \\ ${ }^{1}$ Department of Breast Surgery, Beijing Shijitan Hospital, Capital Medical University, Beijing, China; ${ }^{2}$ Department of Clinical Laboratory, Beijing \\ Shijitan Hospital, Capital Medical University, Beijing Key Laboratory of Urinary Cellular and Molecular Diagnostics, Beijing, China \\ Correspondence to: Man Zhang. Department of Clinical Laboratory, Beijing Shijitan Hospital, Capital Medical University, Beijing Key Laboratory of Urinary \\ Cellular and Molecular Diagnostics. No. 10 Tieyi Road, Yangfangdian Street, Haidian District, Beijing 100038, China. Email: zhangman@bjsjth.cn; \\ Yanping Li. Department of Breast Surgery, Beijing Shijitan Hospital, Capital Medical University. No. 10 Tieyi Road, Yangfangdian Street, Haidian \\ District, Beijing 100038, China. Email: liyanping671102@163.com.
}

\begin{abstract}
Breast cancer is one of the most common malignant tumors in woman. Ovarian and pelvic metastasis in breast cancer are very rare, and the prognosis is often poor. Lacking typical clinical manifestation, misdiagnose is common. We report a case about a middle-aged postmenopausal woman with complaints of finding a left breast mass and irregular vaginal bleeding. Through ultrasound-guided coreneedle biopsy of the masses, the patient's final diagnosis was breast cancer with ovarian and pelvic metastasis. The patient received combinations of chemotherapy (intravenous infusion or peritoneal perfusion), radical surgery (gynecological operation and modified radical mastectomy), radiation and endocrine therapy. Fortyeight months follow-up showed that the patient is generally in good condition and had no signs of local recurrence or metastasis. Invasive ductal carcinoma (IDC) of the breast with unilateral ovarian and pelvic metastasis is rare. It is not easy to differentiate from primary ovarian malignant tumor, and the prognosis is generally poor. We chose a combination chemotherapy regimen sensitive to both breast and ovarian tumors for the patient. The therapeutic effect is remarkable. Only a small number of tumor cells were found in pelvic and ovarian tumors. The patient's breast tumors reached pathological complete remission (pCR), which might be the reason for the patient's good prognosis.
\end{abstract}

Keywords: Breast cancer; ovarian metastasis; pelvic metastasis; case report

Submitted May 08, 2020. Accepted for publication Oct 26, 2020.

doi: $10.21037 /$ tcr-20-2031

View this article at: http://dx.doi.org/10.21037/tcr-20-2031

\section{Introduction}

Breast cancer is one of the most common malignant tumors and one of the leading causes of female cancer death (1). Breast cancer can metastasize to different organs, including lungs, liver, bone and brain, etc. (2). Metastatic breast cancer to the ovary, although less frequent than the above metastatic sites, is also quite common (3). The probability of ovarian metastasis in breast cancer is $3-30 \%$. The ovarian metastasis mostly occurs in the advanced stage of breast cancer, and the prognosis is generally poor (4). Compared with the ovary, pelvic metastasis of breast cancer is less common. Here, we report a case of breast cancer with ovarian and pelvic metastasis, and the therapeutic effect is excellent. We present the following article in accordance with the CARE reporting checklist (5) (available at http:// dx.doi.org/10.21037/tcr-20-2031).

\section{Case presentation}

A 50-year-old female presented in Beijing Shijitan Hospital in October 2015, with complaints of finding a left breast mass for one month and irregular vaginal bleeding for three days. She has no family history of breast cancer. Previously in 2008, the patient was diagnosed with right side breast cancer and underwent a right-side radical mastectomy in 


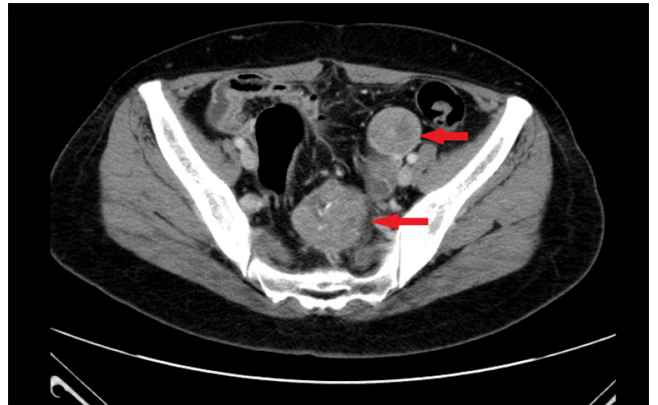

Figure $1 \mathrm{CT}$ scan of pelvic (before treatment). The arrows indicate lesions in the upper regions of the uterus and left adnexal regions.

the treatment of this. The pathology report showed that it was triple-negative breast cancer and Ki-67 was not clear. The adjuvant treatment plan was not clear. She had a regular reexamination and found no abnormality, but the results were not clear. The patient has been menopausal for four years.

Physical examination showed that the patient's right breast was absent, the left breast's skin and nipple were normal, without redness, edema and nipple discharge. Three hard and indistinct masses could be found in the upper part of the left mammary gland. They were $2.0 \mathrm{~cm} \times 2.1 \mathrm{~cm}, 1.5 \mathrm{~cm} \times 1.4 \mathrm{~cm}$ and $4.0 \mathrm{~cm} \times 4.1 \mathrm{~cm}$ in size from the outer upper quadrant to the inner upper quadrant. Axillary lymph nodes and supraclavicular lymph nodes were not touched.

Ultrasound indicated multiple hypoechoic masses on the left breast $(2.1 \mathrm{~cm} \times 1.5 \mathrm{~cm}, 2.2 \mathrm{~cm} \times 1.7 \mathrm{~cm}, 1.9 \mathrm{~cm} \times$ $2.2 \mathrm{~cm}$ ) and suspicious metastatic lymph nodes were also found in the same armpit; a hypoechoic mass was seen on the left adnexal regions $(5.6 \mathrm{~cm} \times 3.4 \mathrm{~cm})$, possible metastasis was considered, and the other hypoechoic mass was seen next to the left lower abdomen $(7.2 \mathrm{~cm} \times 3.0 \mathrm{~cm})$, lymph node metastasis was also suspected. CT scan of pelvic revealed multiple lesions in the upper regions of the uterus $\left(4.5 \times 4.3 \times 4.9 \mathrm{~cm}^{3}\right)$ and left adnexal regions $\left(4.8 \times 3.5 \times 7.0 \mathrm{~cm}^{3}\right)$ (Figure 1). Serum tumor markers level showed an evident elevation, Ca-125: $711.8 \mathrm{U} / \mathrm{mL}$ (normal range, 0-35 U/mL); Ca-153: 155.2 U/mL (normal range, 0-31.3 U/mL).

The patient underwent core-needle biopsy for both breast and pelvic masses (the diagnostic methods and treatment procedures is shown in Figure 2). Pathological diagnosis of the breast biopsy sample revealed an invasive ductal carcinoma (IDC) grade 3. Immunohistochemical analysis indicated estrogen receptor (ER) positive (15\%), progesterone receptor (PR) negative, human epidermal growth factor receptor 2 (HER2) negative and Ki-67 positive (90\%) (Figure 3). Immunohistochemical results were completely different from those of right breast cancer in 2008 , so the left breast tumor was considered as primary IDC. Pathological diagnosis of the pelvic mass (included both the left adnexal regions mass and the left lower abdomen mass) biopsy sample showed metastatic IDC, ER positive (99\%), PR positive (3\%), HER2 negative and Ki67 positive (40\%) (Figure 4). BRCA1 and BRCA2 genes test was negative.

The present authors discussed the case at their multidisciplinary breast cancer conference. The diagnosis of this patient was left breast cancer, ovarian and pelvic metastasis, $\mathrm{T}_{2} \mathrm{~N}_{0} \mathrm{M}_{1}$, stage IV. The common view was obtained that the best approach for this patient was to start with chemotherapy due to the advanced stage. Patients with breast cancer are usually recommended to use taxane-containing drugs for chemotherapy, and patients with ovarian malignant tumors are recommended to use platinum-containing drugs for chemotherapy $(6,7)$. Therefore, we have formulated a chemotherapy regimen of taxane combined with carboplatin (TP) for this patient. Then the patient began a regimen of docetaxel $\left(75 \mathrm{mg} / \mathrm{m}^{2}, \mathrm{q} 3 \mathrm{w}\right.$ ) combined with carboplatin (AUC 4-6, peritoneal perfusion or intravenous infusion, $q 3 \mathrm{w}$ ). After three cycles of chemotherapy, the tumors were significantly reduced on re-examination for both left breast and pelvic sites (Table 1). Then, the operation was undergone in the gynecology department, including hysterectomy, oophorectomy, pelvic lymph node dissection, paraaortic lymph node dissection, pelvic adhesiolysis, and omentectomy after five cycles chemotherapy of TP (peritoneal perfusion for carboplatin) (Figure 5). The postoperative pathology report showed that it was poorly differentiated cancer tissue with multifocal necrosis and the source of tissue was difficult to judge. Except for WT-1 positive, immunohistochemical results and the morphology of tumor cells were consistent with those of left primary breast cancer, and there was no transition between the tumor and ovarian tissue. Therefore, pathologists believed that the tumor was metastasized from left breast cancer (Figure 6). The patient received another cycle of TP chemotherapy (intravenous infusion for carboplatin), and subsequently modified radical mastectomy for the left side of the breast was performed. Pathology showed that no cancer 


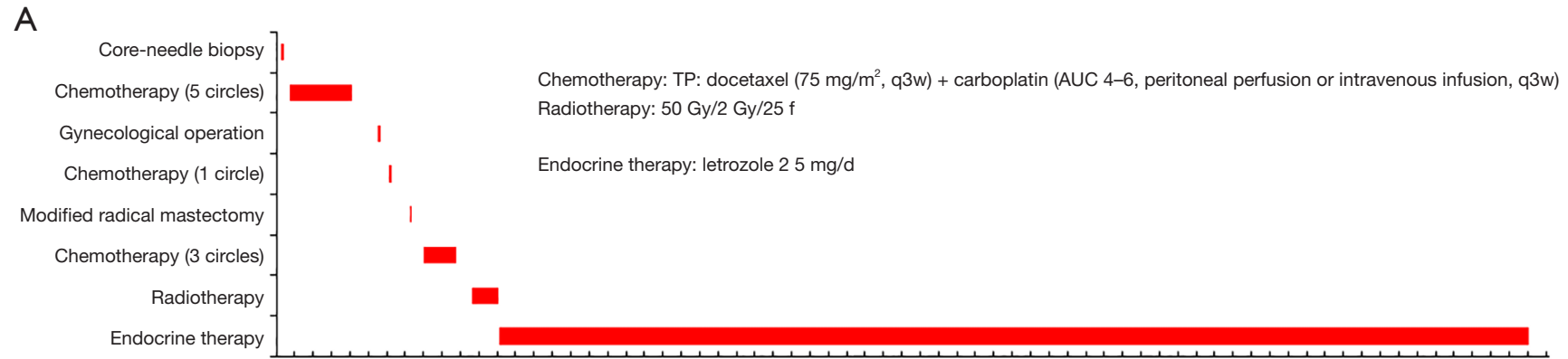

2015/10/23 2016/2/20 2016/6/19 2016/10/17 2017/2/14 2017/6/14 2017/10/12 2018/2/9 2018/6/9 2018/10/7 2019/2/4 2019/6/4 2019/10/2 2020/1/30

B

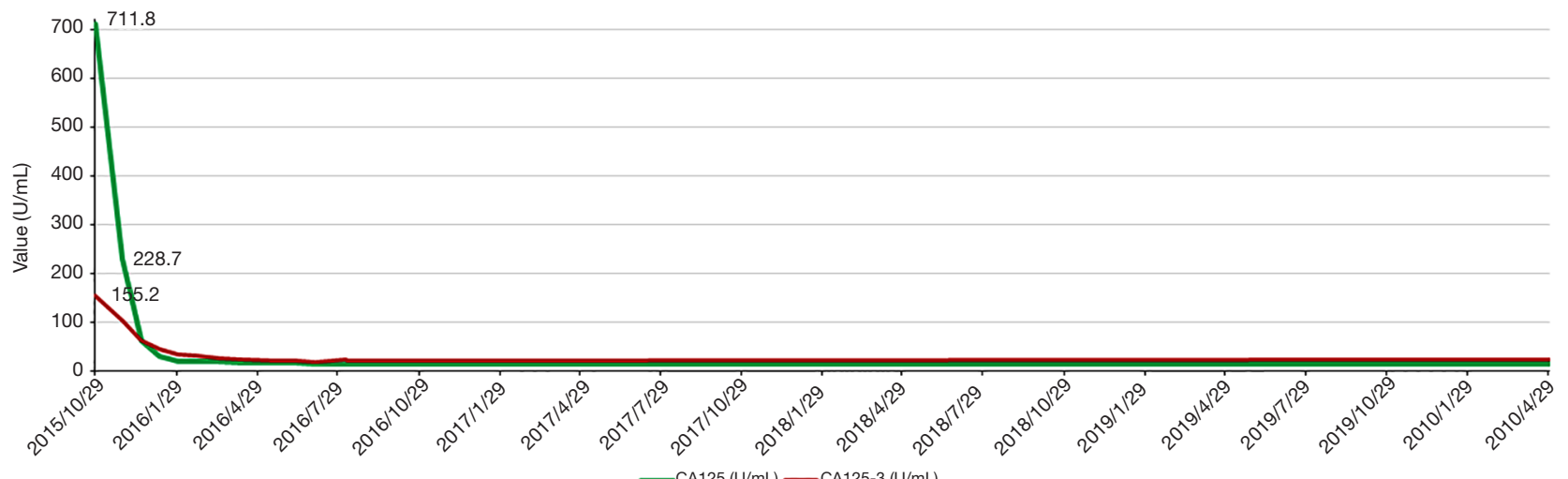

Figure 2 Key treatment methods (A) and tendency chart of tumor markers (B).
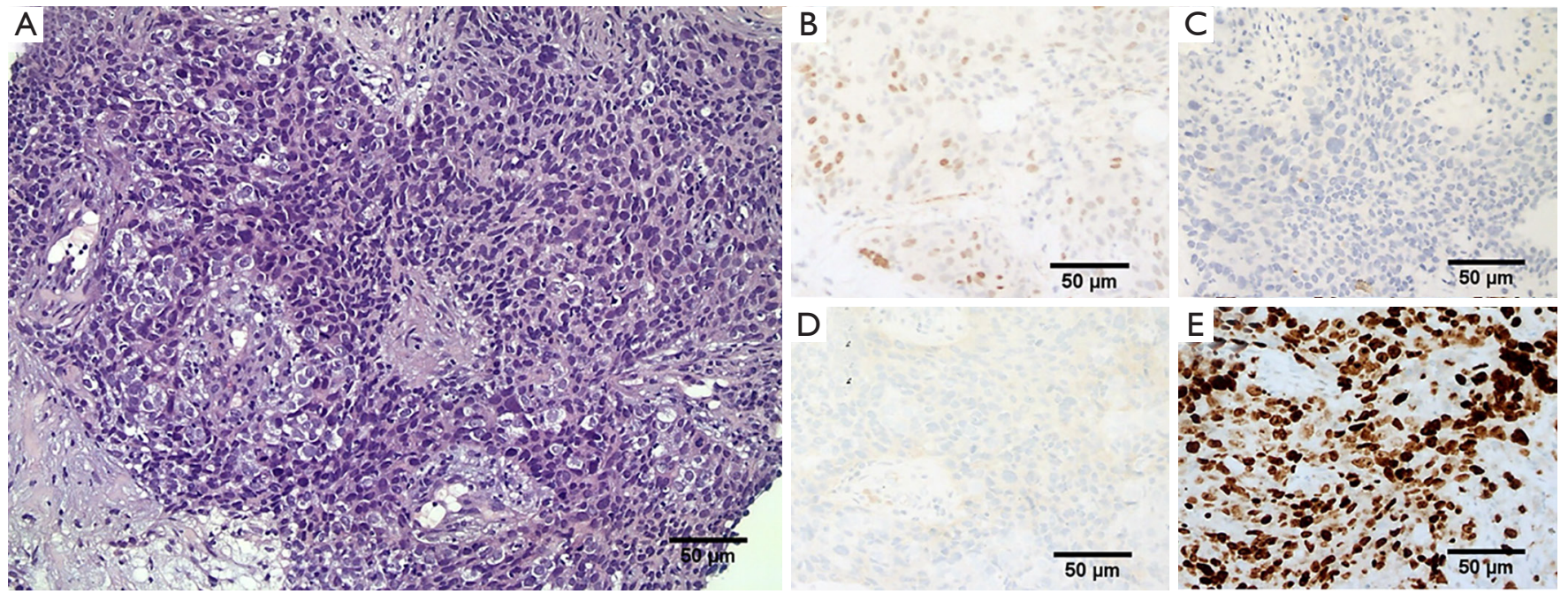

Figure 3 Pathology of breast core-needle biopsy. (A) Invasive ductal carcinoma grade $3(\times 200$, H\&E stain); (B) ER positive (15\%) ( $\times 200$, IHC); (C) PR negative (×200, IHC); (D) HER2 negative (×200, IHC); (E) Ki-67 90\% (×200, IHC). ER, estrogen receptor; PR, progesterone receptor. 

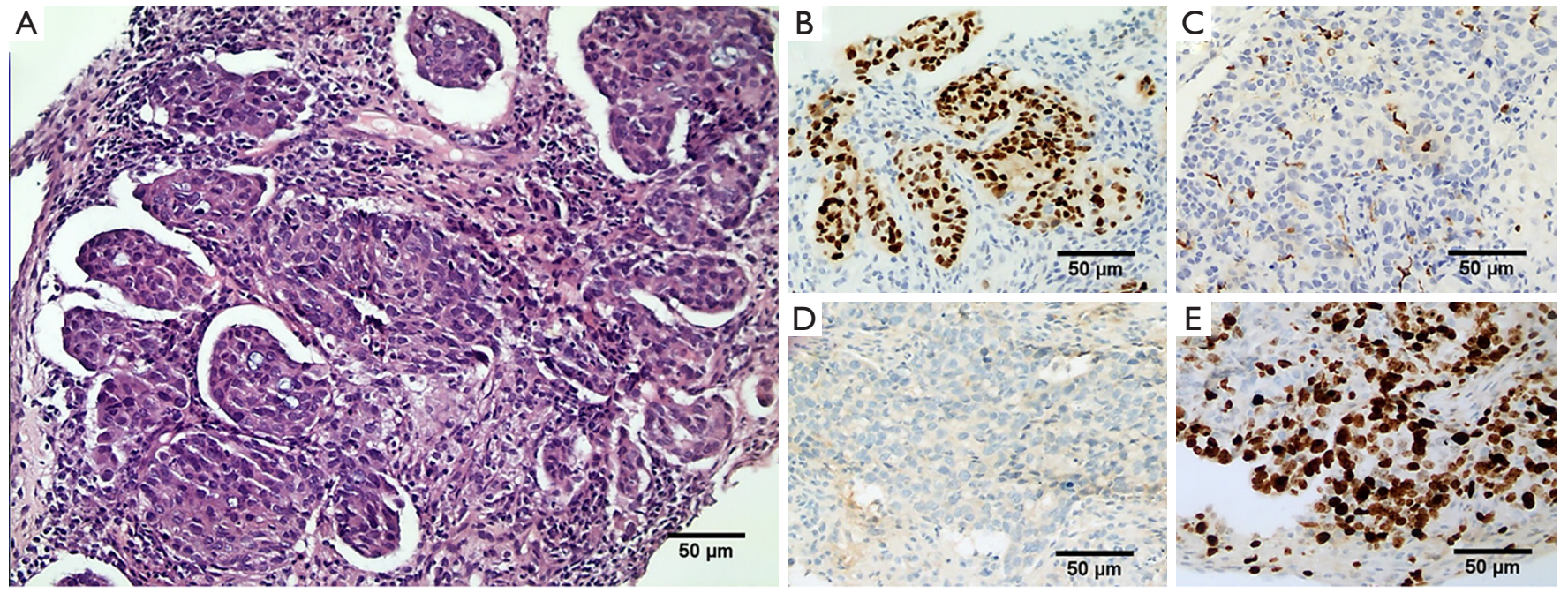

Figure 4 Pathology of pelvic mass core-needle biopsy. (A) Metastatic of invasive ductal carcinoma ( $\times 200$, H\&E stain); (B) ER positive (99\%) ( $\times 200$, IHC); (C) PR positive (3\%) (×200, IHC); (D) HER2 negative ( $\times 200$, IHC); (E) Ki-67 40\% ( $\times 200$, IHC). ER, estrogen receptor; PR, progesterone receptor.

Table 1 The effect of chemotherapy

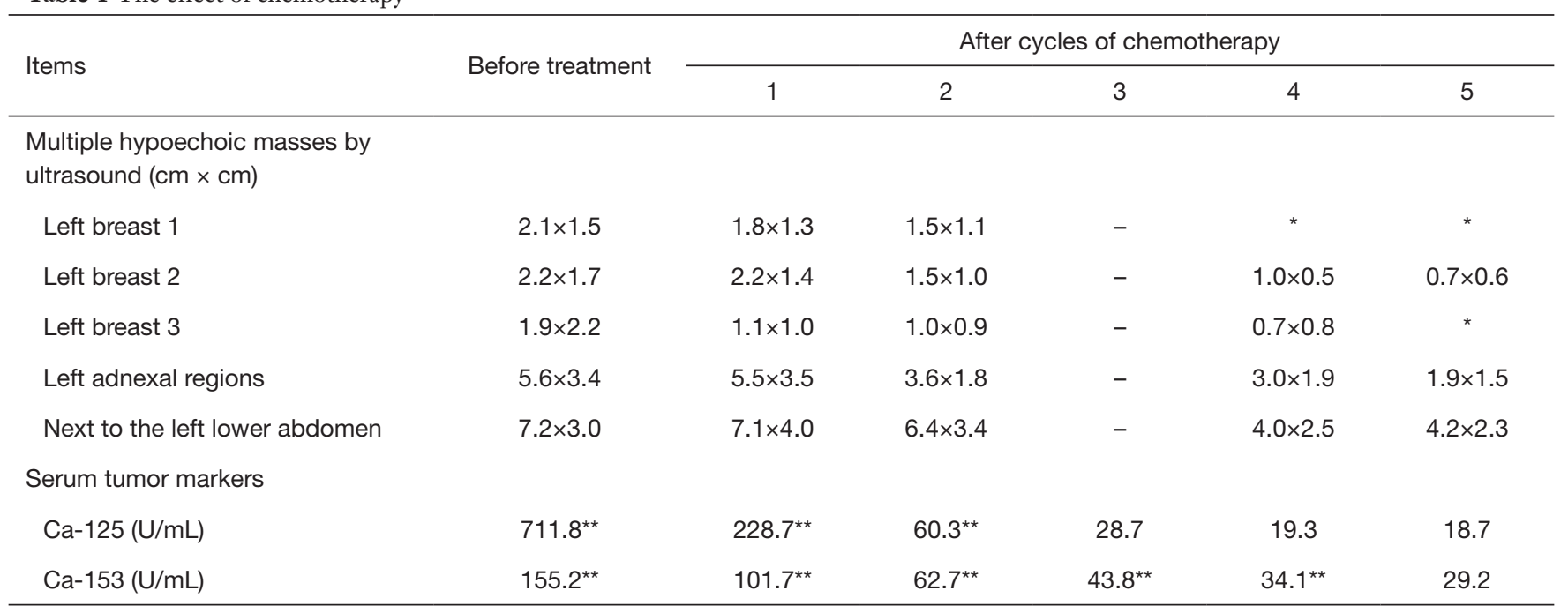

*, was not detected; ${ }^{\star \star}$, higher than the normal value.

cell was found after extensive sampling. Chemotherapy was completely effective (G5), and all axillary lymph modes were negative. The patient continued to receive three cycles of TP chemotherapy after breast surgery, and then accepted chest wall radiotherapy (50 Gy/2 Gy/25 f). After that, the patient has been taking oral letrozole endocrine therapy to date. The patient's compliance with the treatment is good, and she can insist on completing the reexamine on time.
At present, forty-eight months follow-up shows that the patient is generally in good condition and had no signs of local recurrence or metastasis. Disease-free survival (DFS) has been forty-eight months.

All procedures performed in studies involving human participants were in accordance with the ethical standards of the institutional and/or national research committee(s) and with the Helsinki Declaration (as revised in 2013). Written 
informed consent was obtained from the patient.

\section{Discussion}

Breast cancer is the leading cause of cancer in women and ranks sixth among all cancer deaths in China (8). The most common sites of metastasis for breast cancer include the liver, lungs, bones, and brain. Breast cancer metastasized to other sites is relatively rare (2).

The probability of ovarian metastasis in breast cancer is $3-30 \%$ (4). Ovarian metastasis is more likely to occur in premenopausal breast cancer women. The median age at the time of diagnosis was 36.5 years (9). It may be related to

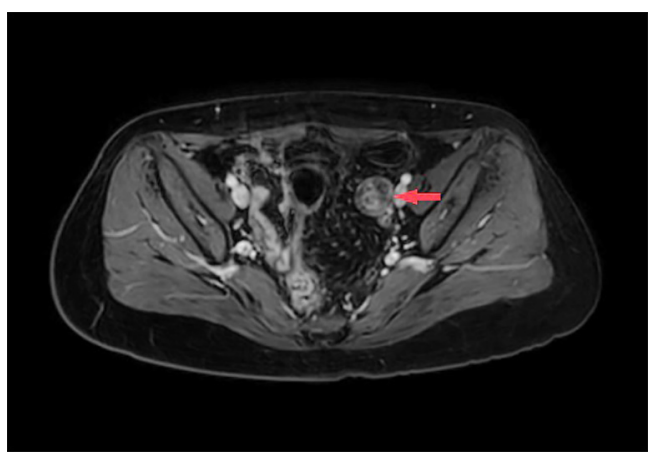

Figure 5 MRI of pelvic (after five circles chemotherapy). The arrow indicates left adnexal regions lesions. factors such as ovarian blood supply and lymphatic drainage are relatively abundant. Malignant tumor cells can be involved in the ovary through blood, lymphatic, peritoneal and abdominal cavity implantation or direct spread; premenopausal women have high estrogen levels, and a large number of estrogens activate cancer cells, making them prone to ovarian metastasis; the mutation of $B R C A 1$ or $B R C A 2$ gene can cause both breast cancer and primary or metastatic ovarian cancer (10). According to reports in the literature, ovarian metastasis of breast cancer is more common in bilateral ovarian metastasis after breast lobular carcinoma, and more often accompanied by extensive metastasis of pelvic or abdominal cavity (11). IDC of the breast with unilateral ovarian and pelvic metastasis is rare.

Ovarian metastasis of breast cancer is common in patients with advanced breast cancer. Before the occurrence of ovarian metastasis, multiple systemic metastases, such as bone metastasis, liver metastasis, lung metastasis are often accompanied by extensive intraperitoneal implantation. Only a few patients showed gynecological endocrine symptoms such as vaginal bleeding, so ovarian metastasis is often misdiagnosed as primary ovarian cancer or missed diagnosis (12). This patient was a postmenopausal woman, and found opposite breast masses with vaginal bleeding. Multiple masses could be touched in the upper quadrant of the left breast and the left adnexal area as well. No metastasis was found in other sites except pelvic and left
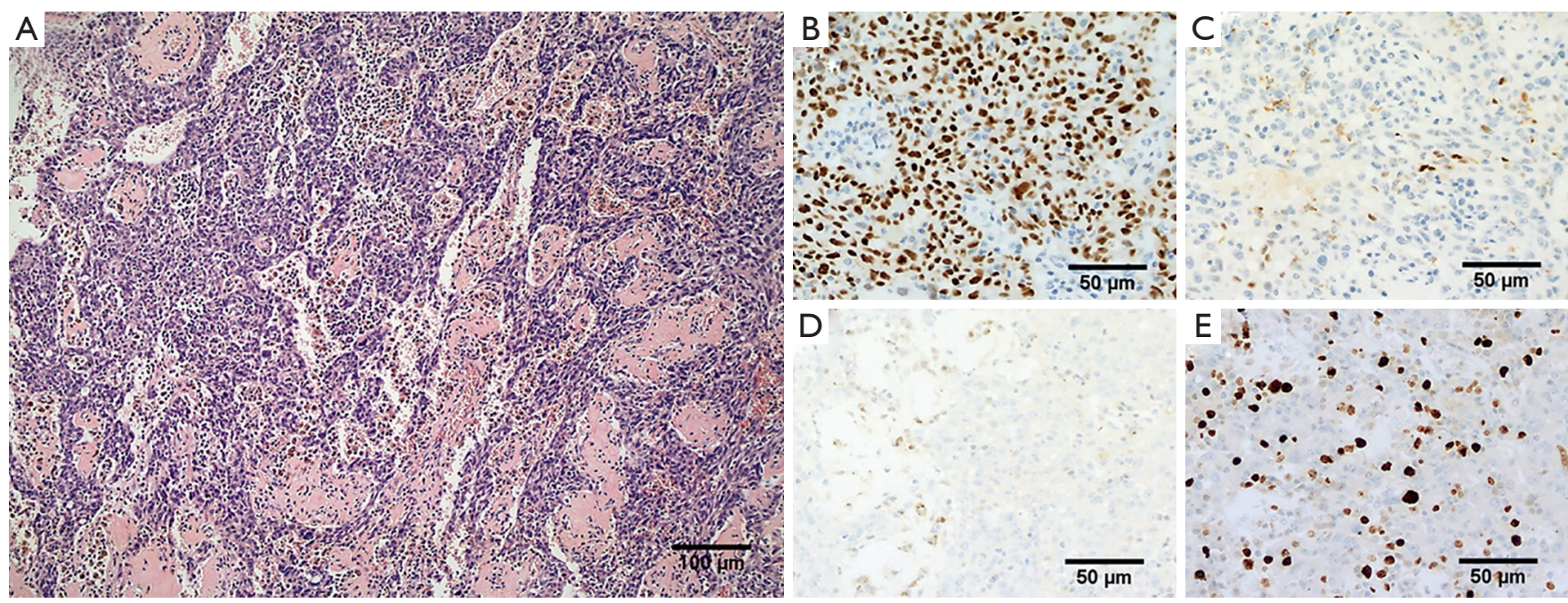

Figure 6 Postoperative pathology of pelvic mass. (A) Metastatic of invasive ductal carcinoma $(\times 100$, H\&E stain); (B) ER positive (80\%) (×200, IHC); (C) PR positive (5\%) (×200, IHC); (D) HER2 negative (×200, IHC); (E) Ki-67 20\% (×200, IHC). ER, estrogen receptor; PR, progesterone receptor. 
adnexal masses. Bruchim indicated Ca-125 levels could accurately discriminate primary ovarian cancer from secondary ovarian tumors (13). Ca-125 >170 U/mL can be used as an auxiliary diagnostic index for discriminating Primary ovarian cancer against secondary one (13). In this case, Ca-125: 711.8 U/mL and Ca-153: $155.2 \mathrm{U} / \mathrm{mL}$, both of them were significantly increased at the initial examination, which led to the possibility of primary ovarian cancer with breast cancer at the initial diagnosis.

Studies showed that IDC would metastasize more commonly to the lung, pleura, and central nervous system, and invasive lobular carcinoma would metastasize more frequently to the bone, peritoneum, gastrointestinal and genitourinary systems, skin, and other unusual sites (14). Winn et al. indicated that invasive lobular carcinoma recurred more often in the abdominal cavity than IDC (24.3\% vs. $4.1 \%$ ) (11). Ovarian metastasis of breast cancer is characterized by premenopausal hormone receptor-positive young women, mostly invasive lobular carcinoma, and more common on both sides (15). Patients with a history of breast cancer are more likely to develop primary or metastatic ovarian cancer, and breast cancer patients with $B R C A 1$ or $B R C A 2$ genes mutations are associated with a higher risk (16). However, this patient was a triple-negative IDC on the right breast in 2008 and a hormone receptor-positive IDC on the left breast in 2015 without breast cancer family history and BRCA mutation. The results of the pelvic mass biopsy showed that the pathological type was much similar to the left side of breast cancer, and both were ER, PR positive, HER2 negative. Pathologists considered that there is no transition between tumors and ovaries and fallopian tubes. Therefore, the conclusion is that pelvic and ovary cancers metastasize from the left primary breast cancer.

The mechanism of ovarian and pelvic metastasis in breast cancer is still unclear. It may be related to the ovarian blood supply, secretion of large amounts of estrogens to activate cancer cells, and most of them occur after breast cancer surgery (median metastasis time is 11.5-104 months) (17). After three years of menopause, this patient was diagnosed with primary breast cancer and ovarian and pelvic metastases at the same time. No other abdominal organs or extensive lymph node metastases were found, which is rare in clinic. Ovarian metastasis of breast cancer often indicates a poor prognosis. Surgical removal of lesions can prolong survival time when other prognostic factors are certain. For hormone receptor-positive patients, at least bilateral ovariectomy is recommended (15). In this patient case, the initial diagnosis was stage IV breast cancer in 2015 and chemotherapy was first administered. For ovarian and pelvic masses, carboplatin intraperitoneal chemotherapy was administered while docetaxel intravenous chemotherapy was given. After chemotherapy, postoperative pathology showed pathological complete remission (pCR) of breast tumors, and only a small amount of tumor tissue could be found in pelvic and ovarian sites. There was no metastasis in omentum, abdominal and pelvic lymph nodes and other tissues. At present, the patient has been followed up for forty-eight months. The general condition of the patients is good. No signs of recurrence or metastasis have been found in all examinations. The patient is satisfied with the treatment effect. After chemotherapy, the primary lesions of breast, pelvic and ovarian metastases all had significant effects, and radical resection of R0 was completed, which could be potential reasons for good therapeutic effect in the case.

Ovarian metastasis in breast cancer is rare. This case describes a postmenopausal woman with breast cancer, ovarian and pelvic metastases. We chose a combination chemotherapy regimen sensitive to both breast and ovarian tumors for the patient. The therapeutic effect is remarkable. Only a small number of tumor cells were found in pelvic and ovarian tumors. The patient's breast tumors reached $\mathrm{pCR}$ which might be the reason for the patient's good prognosis.

\section{Acknowledgments}

The authors thank the Department of Pathology and Department of Imaging for all the technical assistance.

Funding: The authors disclosed receipt of the following financial support for the research, authorship, and/or publication of this article: This work was supported by Enhancement Funding of Beijing Key Laboratory of Urinary Cellular Molecular Diagnostics, Code: 2019-JS02, and Beijing Municipal Administration of Hospitals' Ascent Plan, Code: DFL20150701.

\section{Footnote}

Reporting Checklist: The authors have completed the CARE reporting checklist. Available at http://dx.doi.org/10.21037/ tcr-20-2031

Peer Review File: Available at http://dx.doi.org/10.21037/tcr20-2031 
Conflicts of Interest: All authors have completed the ICMJE uniform disclosure form (available at http://dx.doi. org/10.21037/tcr-20-2031). The authors have no conflicts of interest to declare.

Ethical Statement: The authors are accountable for all aspects of the work in ensuring that questions related to the accuracy or integrity of any part of the work are appropriately investigated and resolved. All procedures performed in studies involving human participants were in accordance with the ethical standards of the institutional and/or national research committee(s) and with the Helsinki Declaration (as revised in 2013). Written informed consent was obtained from the patient.

Open Access Statement: This is an Open Access article distributed in accordance with the Creative Commons Attribution-NonCommercial-NoDerivs 4.0 International License (CC BY-NC-ND 4.0), which permits the noncommercial replication and distribution of the article with the strict proviso that no changes or edits are made and the original work is properly cited (including links to both the formal publication through the relevant DOI and the license). See: https://creativecommons.org/licenses/by-nc-nd/4.0/.

\section{References}

1. DeSantis CE, Ma J, Goding Sauer A, et al. Breast cancer statistics, 2017, racial disparity in mortality by state. CA Cancer J Clin 2017;67:439-48.

2. Kasper DL, Fauci AS, Hauser SL, et al. Breast cancer. Harrison's manual of medicine, New York: McGraw-Hill Education; 2016; vol. 19e.

3. Yadav BS, Sharma SC, Robin TP, et al. Synchronous primary carcinoma of breast and ovary versus ovarian metastases. Semin Oncol 2015;42:e13-e24.

4. Turan T, Aykan B, Koc S, et al. Analysis of metastatic ovarian tumors from extragenital primary sites. Tumori 2006;92:491-5.

5. Gagnier JJ, Kienle G, Altman DG, et al. The CARE Guidelines: Consensus-based Clinical Case Reporting Guideline Development. Glob Adv Health Med

Cite this article as: Feng Y, Zhao X, Lv S, Xiong B, Li Y, Zhang M. Long disease-free survival (48 months) in a breast cancer patient with ovarian and pelvic metastasis-a case report. Transl Cancer Res 2020;9(12):7669-7675. doi: 10.21037/tcr-20-2031
2013;2:38-43.

6. Expert Group on the Clinical Practice of Taxanes for the Treatment of Breast Cancer. Expert consensus on the clinical practice of taxanes for the treatment of breast cancer. Zhonghua Zhong Liu Za Zhi 2020;42:161-9.

7. Casado A, Callata HR, Manzano A, et al. Trabectedin for reversing platinum resistance and resensitization to platinum in patients with recurrent ovarian cancer. Future Oncol 2019;15:271-80.

8. Fan L, Strasser-Weippl K, Li JJ, et al. Breast cancer in China. Lancet Oncol 2014;15:e279-e289.

9. Peters IT, van der Steen MA, Huisman BW, et al. Morphological and phenotypical features of ovarian metastases in breast cancer patients. BMC Cancer 2017;17:206.

10. Ternovoĭ SK, Matkhev S, Meladze NV, et al. Vestn Rentgenol Radiol 2012;(5):16-21.

11. Winn JS, Baker MG, Fanous IS, et al. Lobular Breast Cancer and Abdominal Metastases: A Retrospective Review and Impact on Survival. Oncology 2016;91:135-42.

12. Abd El hafez A, Monir A. Diagnostic spectrum of ovarian masses in women with breast cancer; magnetic resonance imaging: histopathology correlation. Ann Diagn Pathol 2013;17:441-7.

13. Bruchim I, Ben-Harim Z, Piura E, et al. Preoperative clinical and radiological features of metastatic ovarian tumors. Arch Gynecol Obstet 2013;288:615-9.

14. Oliveira NC, Gomig TH, Milioli HH, et al. Comparative proteomic analysis of ductal and lobular invasive breast carcinoma. Genet Mol Res 2016. doi: 10.4238/ gmr.15027701.

15. Pimentel C, Becquet M, Lavoue V, et al. Ovarian Metastases from Breast Cancer: A Series of 28 Cases. Anticancer Res 2016;36:4195-200.

16. Bergfeldt K, Rydh B, Granath F, et al. Risk of ovarian cancer in breast-cancer patients with a family history of breast or ovarian cancer: a population-based cohort study. Lancet 2002;360:891-4.

17. Tserkezoglou A, Kontou S, Hadjieleftheriou G, et al. Primary and metastatic ovarian cancer in patients with prior breast carcinoma. Pre-operative markers and treatment results. Anticancer Res 2006;26:2339-44. 